\title{
Chronic restraint stress induces esophageal fibrosis with enhanced oxidative stress in a murine model
}

\author{
MAIMAITI YISIREYILI ${ }^{1,2}$, WUBULIKASIMU WULAMU ${ }^{1,2}$, AIKEBAIER AILI $^{1,2}$, YILIANG LI $^{2}$, \\ AZIGULI ALIMUJIANG ${ }^{3}$, ALIYEGULI AIPIRE ${ }^{1}$, MAIMAITIAILI AIZEZI $^{4}$, WEIMIN ZHANG ${ }^{4}$, \\ ZHENGYI CAO $^{2}$, ABULAJIANG MIJITI $^{2}$ and KELIMU ABUDUREYIMU ${ }^{1,2}$ \\ ${ }^{1}$ Research Institute of General and Minimally Invasive Surgery; Departments of ${ }^{2}$ Minimally Invasive \\ Surgery, Hernia and Abdominal Wall Surgery, ${ }^{3}$ Obstetrics and Gynecology Clinic, and ${ }^{4}$ Cardiac Surgery, \\ People's Hospital of Xinjiang Uygur Autonomous Region, Urumqi, Xinjiang 830001, P.R. China
}

Received August 8, 2018; Accepted April 11, 2019

DOI: $10.3892 /$ etm.2019.7669

\begin{abstract}
Although the underlying mechanism of stress remains unknown, it has been associated with the pathophysiology of gastroesophageal reflux diseases, the development of which appear to be accelerated by oxidative stress and fibrosis. The aim of the current study was to investigate the effect of chronic restraint stress on esophageal oxidative stress and fibrosis, as well as the impact of oxidative stress in a murine model whereby 8 -week old C57BL/6J male mice were subjected to intermittent chronic restraint stress for a two-week period. The current study demonstrated that chronic restraint stress significantly reduced the body weight of mice compared with the control group. Although chronic restraint stress did not significantly alter the levels of triglycerides or cholesterol, free fatty acid concentration was significantly increased compared with the control group. Furthermore, chronic restraint stress significantly upregulated the expression levels of several fibrotic biomarkers including collagen type I, transforming growth factor $\beta-1, \alpha$-smooth muscle actin and SMAD-3 compared with the control group. In addition, the expression levels of the reactive oxygen species (ROS) NADPH oxidase-4 and malondialdehyde were significantly increased, while the expression levels of nuclear factor erythroid 2-related factor 2 and heme oxygenase-1 were significantly decreased in esophageal tissue from mice in the chronic restraint stress group compared with the control group. In conclusion, chronic restraint stress may induce esophageal fibrosis by
\end{abstract}

Correspondence to: Professor Kelimu Abudureyimu, Department of Minimally Invasive Surgery, Hernia and Abdominal Wall Surgery, People's Hospital of Xinjiang Uygur Autonomous Region, 91 Tianchi Road, Urumqi, Xinjiang 830001, P.R. China E-mail:klm6075@163.com

Key words: stress, oxidative stress, anti-oxitative proteins, fibrotic biomarkers accumulating ROS and increasing fibrotic gene expression in a murine model.

\section{Introduction}

Stress is triggered when various psychological, physiological or environmental stressors induce a state of threatened homeostasis (1). Stress has be en identified as a risk factor in $75-90 \%$ of all diseases, including those that lead to morbidity and mortality (2). The production of reactive oxygen species (ROS), which are neutralized by enzymatic and non-enzymatic anti-oxidant defense mechanisms, has been recognized as a key mechanism of stress (3). Among the several types of stress (including acute and episodic acute), chronic stress can affect the balance between the production and scavenge of ROS in cells, thereby disrupting metabolic regulation and causing oxidative damage (4). Chronic stress can be classified into several types (including restraint stress and chronic heterotypic stress), with some leading to oxidative stress in several tissues, including the brain, lungs, heart, kidney and liver (5-8).

Gastroesophageal reflux associated tissue fibrosis can lead to esophageal stiffness, reduced esophageal compliance, increased smooth muscle mass and reduced esophageal diameter, leading to smooth muscle dysfunction, esophageal strictures and ultimately a decreased quality of life (9). Some studies have demonstrated that chronic stress can regulate the expression of gene-regulating anti-oxidant systems and NADPH oxidase (NOX), a major driver of ROS production in various types of cells $(10,11)$. NOX-induced ROS have been identified as main sources of oxidative stress, which can accelerate the progression of various fibrotic diseases, including skin fibrosis (12), idiopathic pulmonary fibrosis (13), liver fibrosis (14), cardiovascular fibrosis (15) and kidney fibrosis (16).

Previous studies reported that the psychological-induced oxidative stress can be observed in different cells or tissues, including white adipose and intestinal tissues $(17,18)$. Given the lack of research on esophageal fibrosis, the aim of the current study was to investigate the effect of chronic stress-induced esophageal fibrosis using a chronic restraint stress mouse model. 


\section{Materials and methods}

Experimental animals. A total of 30 male C57BL/6J mice (age, 8 weeks; weight, $25.6 \pm 2.52 \mathrm{~g}$ ) were obtained from the Animal Center of Xinjiang Medical University (Urumqi, China) and used in subsequent experiments. Mice were placed in cages and housed in a viral-pathogen-free facility at the Research Institute of Uygur Pharmaceutics (Urumqi, China) under standard conditions (temperature, $21-25^{\circ} \mathrm{C}$ and humidity, $50 \pm 5 \%$ ) with a $12 \mathrm{~h}$ light/dark cycle. All mice received free access to water and a normal chow diet (Teklad Diet; 18\% fat, $24 \%$ protein, $58 \%$ carbohydrates). All animal experiments were approved by the Animal Care and Use Committee of the People's Hospital of Xinjiang Uygur Autonomous Region (protocol no. KY201803703), and all experimental procedures complied with the Guidelines for the Care and Use of Laboratory Animals published by the National Institute of Health.

Chronic restraint stress protocol. Mice were randomly divided into two groups: A control and a chronic restraint stress group. Mice in the control group were housed in the individual cages and were left undisturbed. Mice in the chronic restraint stress group were subjected to restraint stress using a ventilated plastic $50 \mathrm{ml}$ tube that allowed for a close fit to mice. Subsequently, mice were submitted to immobilization stress for $2 \mathrm{~h}$ per day for period of 14 consecutive days using a self-made restraint device (a $50 \mathrm{ml}$ centrifugal tube with a number of ventilation holes $\sim 5 \mathrm{~mm}$ in diameter and a small hole for the tail) $(17,18)$. During the stress period, mice were not allowed access to food and water. Following chronic restrained stress, mice were maintained in individual cages and allowed free access to food and water. Body weight and food intake were monitored every two days during the stress period.

Sample collection. All mice underwent a $16-18 \mathrm{~h}$ fasting period and were euthanized by intraperitoneal injection of $150 \mathrm{mg} / \mathrm{kg}$ sodium pentobarbital. Blood samples were collected from the inferior vena cava for biological analysis. Esophageal tissue samples were collected to examine chronic restraint stress-induced pathology, as well as the expression levels of specific biological markers.

Histological analysis. Esophageal tissue was collected, weighed, fixed in $10 \%$ formalin for $24 \mathrm{~h}$ at room temperature and dehydrated by a descending series of ethanol at room temperature for $4 \mathrm{~h}$. Tissue samples were embedded in paraffin and cut into $4-\mu \mathrm{m}$-thick sections. Tissue sections were subsequently stained (all at room temperature for $\sim 2.5 \mathrm{~h}$ ) with hematoxylin and eosin (H\&E), Sirus red or Masson's trichrome (MT) and observed under a light microscope (magnification, x200) and imaged using a digital camera (Eclipse E200; Nikon Corporation). Staining was observed in 10 randomly selected fields and analyzed using Adobe Photoshop (Adobe, Inc.) and ImageJ (version 1.62; National Institutes of Health). Histological sections were examined for stress-induced inflammatory changes and scored by a 'blinded' observer for three parameters (19): i) Epithelial damage (0, normal morphology; 1 , mild surface lifting; 2 , intraepithelial separation and surface lifting; and 3, epithelial cell loss to basal cell layer or deeper); ii) submucosal edema ( 0 , normal; 1 , mild focal edema; 2 , moderate diffuse edema; and 3, severe edema); iii) submucosal inflammation ( $0,0-5 /$ high power field (HPF); 1,5-10/HPF; 2, 10-15/HPF; 3, $\geq 15 / \mathrm{HPF}$ ). Each individual score represented the mean of the three sections.

Immunohistochemistry. The streptavidin-biotinylated peroxidase complex method was performed as previously described $(17,18)$. Briefly, esophageal tissue sections were deparaffinized in xylene and rehydrated with a descending ethanol series at room temperature. Endogenous peroxidase activity was inhibited using $0.3 \% \mathrm{H}_{2} \mathrm{O}_{2}$ in methanol for $10 \mathrm{~min}$ in room temperature. Samples were then rinsed with PBS and incubated with $10 \%$ goat normal serum (cat. no. 414322F; Nichirei Biosciences, Inc.) for $30 \mathrm{~min}$ at room temperature. Sections were then treated with the following primary antibodies at $4{ }^{\circ} \mathrm{C}$ overnight: NAPDH oxidase 4 (Nox4; cat. no. ab195524; 1:100; Abcam), malondialdehyde (MDA; cat. no. ab6463; 1:100; Abcam), nuclear factor erythroid 2-related factor 2 (Nrf-2; cat. no. ab62352; 1:100; Abcam), heme oxygenase 1 (HO-1; cat. no. ab13248; 1:100; Abcam), collagen type I (cat. no. ab34710; 1:100; Abcam), transforming growth factor $\beta$-1 (TGF- $\beta-1$; cat. no. sc-130348; 1:100; Santa Cruz Biotechnology, Inc.), $\alpha$-smooth muscle actin (SMA; cat. no.A5228; 1:100; Sigma-Aldrich; Merck KGaA), SMAD-3 (cat. no. \#8685; 1:100; Cell Signaling Technology, Inc.) and F4/80 (cat. no. ab240946; 1:100; Abcam). Sections were subsequently incubated with horseradish peroxidase (HRP)-conjugated anti-rabbit IgG (1:200; cat. no. 414181F; Nichirei Biosciences, Inc.) or HRP-conjugated anti-mouse $\mathrm{IgG}$ secondary antibodies (1:200; cat. no. 414191F; Nichirei Biosciences, Inc.) at room temperature for $30 \mathrm{~min}$. Samples were then rinsed with PBS and treated with peroxidase-conjugated streptavidin (Nichirei Biosciences, Inc.) at $37^{\circ} \mathrm{C}$ for $30 \mathrm{~min}$. Tissue sections were subsequently stained with 3,3-diaminobenzidine tetra-hydrochloride (DAB; Sigma-Aldrich; Merck KGaA) with $0.03 \% \mathrm{H}_{2} \mathrm{O}_{2}$ at room temperature for $15 \mathrm{~min}$ to visualize the localization of Nox4, MDA, Nrf-2, HO-1, collagen type I, TGF $\beta-1, \alpha$-SMA and SMAD-3. esophageal tissue sections were counterstained with methylene green, observed under a light microscope (magnification, $\mathrm{x} 200$ ) and imaged using a digital camera (Eclipse E200; Nikon Corporation). Nox4, MDA, Nrf-2, HO-1, collagen type I, TGF $\beta-1, \alpha$-SMA and SMAD-3 positive cells were observed in 10 randomly selected fields/section and analyzed using Adobe Photoshop (Adobe, Inc.) and quantified using ImageJ (version 1.45S; National Institutes of Health).

Reverse transcription-quantitative PCR (RT- $q P C R)$. Total RNA was extracted from esophageal tissues using TRIzol ${ }^{\circledR}$ reagent (Invitrogen; Thermo Fisher Scientific, Inc.). Total RNA $(1 \mu \mathrm{g})$ was then reverse transcribed into cDNA using the RT system (Qiagen $\mathrm{GmbH}$ ) in accordance with the manufacturer's protocol and qPCR was subsequently performed under the following thermocycling conditions: Initial denaturation for $2 \mathrm{~min}$ at $95^{\circ} \mathrm{C}$, followed by 40 cycles for $12 \mathrm{sec}$ at $95^{\circ} \mathrm{C}$ and $60 \mathrm{sec}$ at $60^{\circ} \mathrm{C}$. The Bio-Rad CFX96 RT-PCR Detection System (Bio-Rad Laboratories, Inc.) and Power SYBR Green PCR Master mix (Applied Biosystems; Thermo Fisher Scientific, Inc.) were utilized for PCR. The primer pairs used for qPCR are presented in Table I. Serial dilutions of a control 
Table I. Primer sequences used in reverse transcription-quantitative PCR.

\begin{tabular}{ll}
\hline Gene & \multicolumn{1}{c}{ Primer sequence (5'-3') } \\
\hline Nox4 & F: CACCTCTGCCTGCTCATTTGG $(\mathrm{bp})$ \\
& R: AGTTGAGGTTCAGGACAGATGC \\
Nrf-2 & F: CGAGATATACGCAGGAGAGGTA AGA \\
& R: GCTCGACAATGTTCTCCAGCTT \\
HO-1 & F: CAGCCCCACCAAGTTCAAAC \\
& R: AGGCGGTCTTAGCCTCTTCTG \\
Collagen type I & F: GGAATGAAAGGGACACAGAGG \\
& R: TAGCACCATCATTTCCACGA \\
TGF- $\beta 1$ & F: GGACTCTCCACCTGCAAGAC \\
& R: GACTGGCGAGCCTTAGTTTG \\
SMAD-3 & F: CATCGAGCCCCAGAGCAATA \\
$\alpha$-SMA & R: GTGGTTCATCTGGTGGTCACT \\
& F: TGCTGACAGAGGCACCACTGAA \\
$\beta$-actin & R: CAGTTGTACGTCCAGAGGCATA \\
& F: TATTGGCAACGAGCGGTTC \\
& R: ATGCCACAGGATTCCATACCC \\
\hline
\end{tabular}

F, forward; R, reverse; Nox4, NAPDH oxidase 4; Nrf-2, nuclear factor erythroid 2-related factor 2; HO-1, heme oxygenase 1 ; TGF- $\beta 1$, transforming growth- $\beta 1 ; \alpha$-SMA, $\alpha$-smooth muscle actin.

cDNA sample were taken and used as the standard curve for each reaction. mRNA levels were quantified using the $2^{-\Delta \Delta \mathrm{Cq}}$ method (20) and normalized to the internal reference gene $\beta$-actin. Each experiment was performed in triplicate.

Western blot analysis. Total protein was extracted from esophageal tissue $(\sim 30 \mathrm{mg})$ using lysis buffer $[65 \mathrm{mmol} / 1$ Tris- $\mathrm{HCl}$ (pH 6.8), 3.3\% SDS, $10 \%$ glycerol, $2.2 \%$ bromophenol blue]. Protein concentration was subsequently determined using a BCA protein assay kit (Pierce; Thermo Fisher Scientific, Inc.). Equal quantities of protein $(50 \mu \mathrm{g})$ were separated via SDS-PAGE on a $10-15 \%$ polyacrylamide gel. The separated proteins were transferred onto polyvinylidene difluoride membranes and blocked for $1 \mathrm{~h}$ at room temperature with 5\% bovine serum albumin (cat. no. 10735078001; Sigma-Aldrich; Merck KGaA) in Tris-buffered saline containing Tween ${ }^{\circledR}-20$ (TBS-T). Membranes were washed with TBS-T, and incubated with the following primary antibodies (all, 1:1,000): Nrf-2, phosphoNrf-2 (cat. no. ab76026; 1:100; Abcam), Kelch-like ECH-associated protein 1 (keap-1; cat. no. PAL648Mu01; Cloud-Clone Corp.), HO-1, collagen type I, TGF $\beta-1, \alpha$-SMA, SMAD-3 and normalized to proliferating cell nuclear antigen (cat. no. M0879; Dako; Agilent technologies, Inc.) and $\beta$-actin (cat. no. \#3700; Cell Signaling Technology, Inc.). Following primary antibody incubation, membranes were further incubated with HRP-conjugated anti-mouse IgG (cat. no. \#7076; Cell Signaling Technology, Inc.) and HRP-conjugated anti-rabbit IgG secondary antibodies (cat. no. \#7074; Cell Signaling Technology, Inc.; each, 1:10,000) for $1 \mathrm{~h}$ at room temperature. Membranes were then washed three times with TBS-T. Protein bands were visualized using the enhanced Chemi-Lumi One System (Nacalai Tesque, Inc.).
ELISA. Plasma samples were taken from all mice and processed as previously described $(17,18)$. Plasma Nox4 (cat. no. SEB924Mu; Cloud-Clone Corp.), MDA (cat. no. KGE013; R\&D Systems, Inc.), total cholesterol (cat. no. ab65390; Abcam), tryglycerides (cat. no. ab178780; Abcam) and free fatty acids (FFA; cat. no. ab65341; Abcam) expression was detected using competitive ELISA kits, according to the manufacturer's protocol.

Statistical analysis. Data presented as the mean \pm standard deviation and were analyzed using GraphPad Prism 5.01 software (GraphPad Software, Inc.). A Student's t-test was performed to analyze the differences between the chronic restraint stress and control groups with SPSS 19 software (IBM, Corp.). One-way analysis of variance followed by a Fisher's protected least significant differences test was performed to analyze the quantitative data collected from both groups. $\mathrm{P}<0.05$ was considered to indicate a statistically significant difference.

\section{Results}

Chronic restraint stress increases fibrotic biomarker expression in esophageal tissue. The expression of fibrotic proteins, including collagen type I, TGF- $\beta 1$, SMAD- 3 and $\alpha$-SMA were examined in esophageal tissue using immunohistochemistry, RT-qPCR and western blotting. Compared with control mice, the expression of fibrotic proteins in stressed mice was predominantly located in the mucosal and epithelial layers of the esophagus (as indicated by arrows; Fig. 1A-D). In addition, chronic restraint stress significantly increased the expression of collagen type I, TGF- $\beta 1$, SMAD- 3 and $\alpha$-SMA in the mucosal and epithelial layers of the esophagus when compared with the 

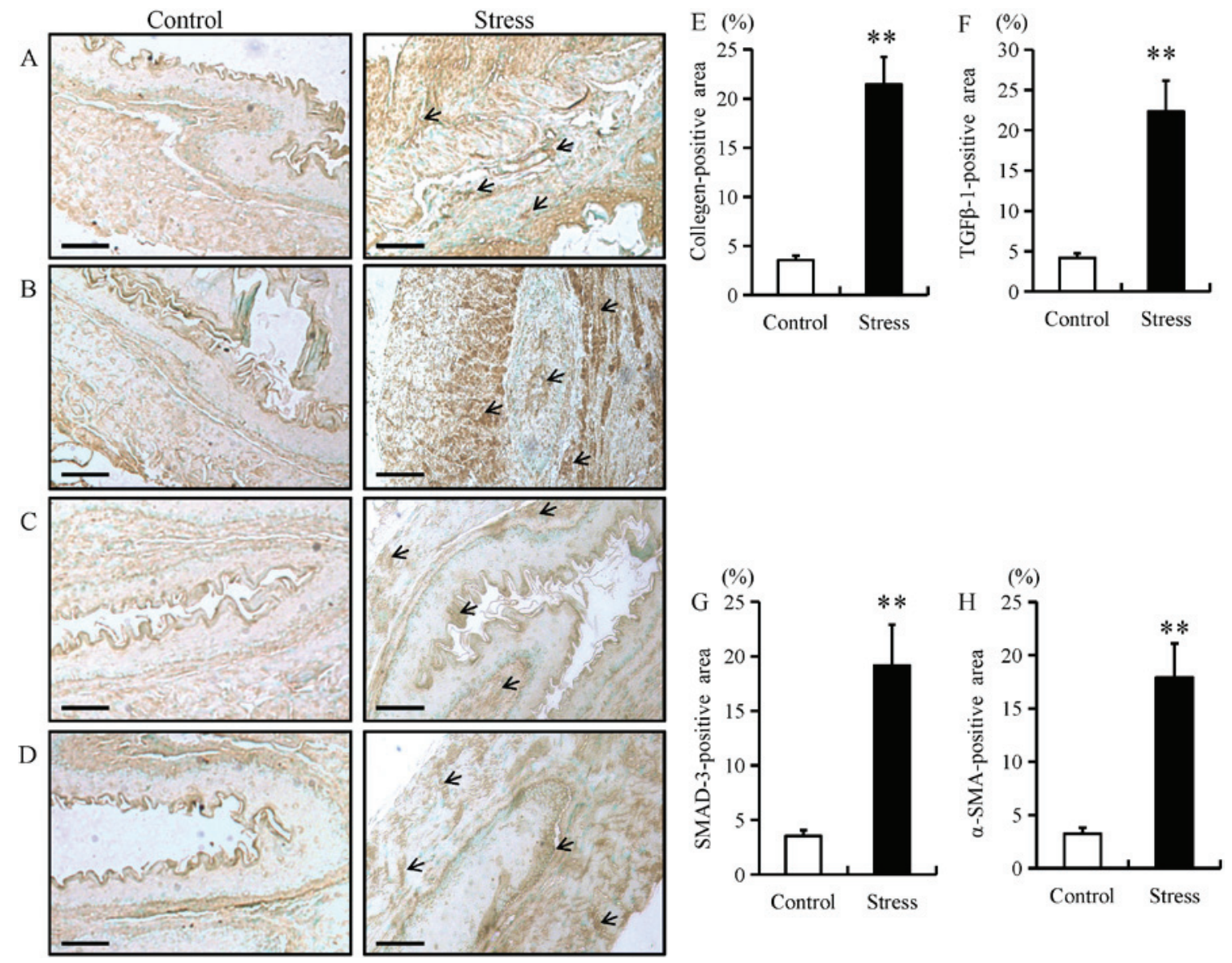

Figure 1. Immunostaining of fibrotic biomarkers in esophageal tissue samples from mice. Immunohistochemistry was performed on esophageal tissue samples from mice in the chronic restraint stress and control groups. Immunostaining for (A) collagen type I, (B) TGF- $\beta 1$, (C) SMAD-3 and (D) $\alpha$-SMA fibrotic markers (magnification, x200; scale bar, $50 \mu \mathrm{m}$ ). Quantification of (E) collagen type I, (F) TGF- $\beta 1$, (G) SMAD-3 and (H) $\alpha$-SMA positive areas. Data presented as the mean \pm standard deviation $(n=15) .{ }^{* *} \mathrm{P}<0.001$ vs. control. TGF- $\beta 1$, transforming growth factor $\beta 1$; SMAD-3, SMAD family member 3 ; $\alpha$-SMA, $\alpha$-smooth muscle actin.

control group (Fig. 1A-H). Furthermore, the mRNA and protein expression of these fibrotic proteins were significantly increased in the esophageal tissue of mice in the chronic restraint stress group compared with the control group (Fig. 2A-E).

Chronic restraint stress induces ROS generation in the esophagus. Chronic stress has been previously reported to trigger ROS production in adipose (18) and colon tissue (21). To determine whether chronic stress triggers the generation of ROS in the esophagus, the expression of Nox4 and MDA were determined using immunohistochemistry in the esophageal tissue of mice. The expression of Nox4 and MDA were predominantly located in the mucosal and epithelial layers of the esophagus (as indicated by arrows; Fig. 3A and B). In addition, chronic restraint stress significantly increased the expression of Nox4 and MDA in the mucosal and epithelial layers of the esophagus (Fig. 3C and D). Furthermore, chronic restraint stress significantly upregulated Nox4 mRNA levels, as well as Nox4 and MDA plasma expression compared with the control group (Fig. 3E-G).

Chronic restraint stress reduces esophageal expression of anti-oxidant proteins. Under normal physiological conditions, Nrf-2 remains in an inactive form in the cytoplasm by Keap1 (22). Nrf-2 can be activated by diverse stimuli, including oxidants, pro-oxidants and antioxidants (22). Both Nrf-2 and its downstream target gene, HO-1, serve as major regulators in the protection against oxidative stress in the esophagus (23). Oxidative stress can damage the function of the epithelial barrier in the gastrointestinal system (24). Thus, to investigate the potential role of $\mathrm{Nrf}-2$ in esophageal fibrosis, the expression of Nrf-2 and HO-1 was examined in in esophageal tissue from mice subjected to chronic restraint stress. The expression of Nrf-2 and HO-1 were predominantly located in the mucosal and epithelial layers of the esophagus (as indicated by arrows; Fig. 4A and B). In addition, chronic restraint stress significantly decreased the expression of Nrf-2 and HO-1 in the mucosal and epithelial layers of the esophagus (Fig. 4C and D). Chronic restraint stress also significantly decreased the mRNA level of Nrf-2 and HO-1 mRNA compared with the control group (Fig. 4E and F). Furthermore, Keap-1, p-Nrf-2, $\mathrm{Nrf}-2$ and HO-1 protein levels were downregulated in both the nucleus and cytoplasm fractions from the chronic restraint stress group compared with the control group (Fig. 4G and H).

Chronic restraint stress induces weight loss and FFA release in mice. Consistent with prior studies $(17,18)$, subjecting the mice to chronic restraint stress for two weeks significantly reduced their body weight compared with the mice in the control group (Table II). In addition, there were no significant 
A

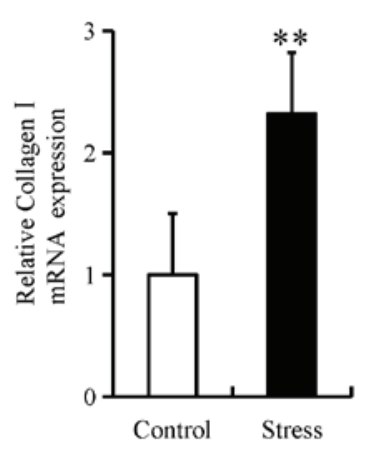

$\mathrm{C}$

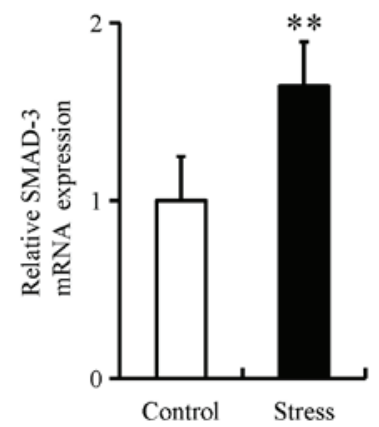

B

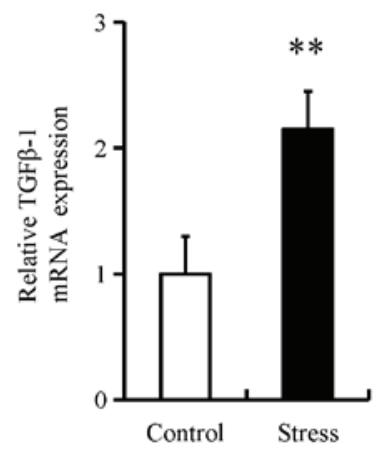

D

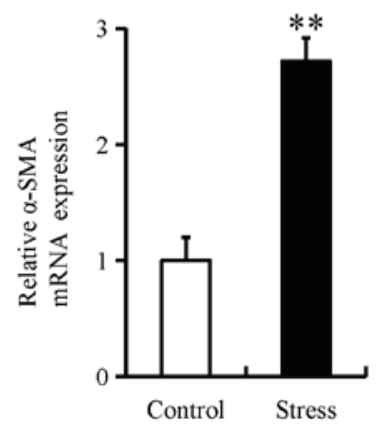

E Control Stress

$\begin{array}{rl}\text { Collagen I } & 220 \mathrm{kDa} \\ \text { TGF } \beta-1 & 25 \mathrm{kDa} \\ \text { SMAD-3 } & 52 \mathrm{kDa} \\ \alpha \text {-SMA } & \\ \beta \text {-actin } & 42 \mathrm{kDa} \\ 45 \mathrm{kDa}\end{array}$

Figure 2. Chronic restraint stress increases esophageal expression of fibrotic biomarkers. The relative mRNA expression levels of (A) collagen type I, (B) TGF- $\beta 1$, (C) SMAD-3 and (D) $\alpha$-SMA was determined by reverse transcription-quantitative PCR in esophageal tissue from mice in the chronic restraint stress and control groups. The relative protein expression level of (E) collagen type I, TGF- $\beta 1$, SMAD-3 and $\alpha$-SMA was determined via western blot analysis of esophageal tissue from mice in the chronic restraint stress and control groups. Data presented as the mean \pm standard deviation $(n=15){ }^{* * *} \mathrm{P}<0.001 \mathrm{vs}$. control. TGF- $\beta 1$, transforming growth factor $\beta 1$; SMAD-3, SMAD family member 3 ; $\alpha$-SMA, $\alpha$-smooth muscle actin.
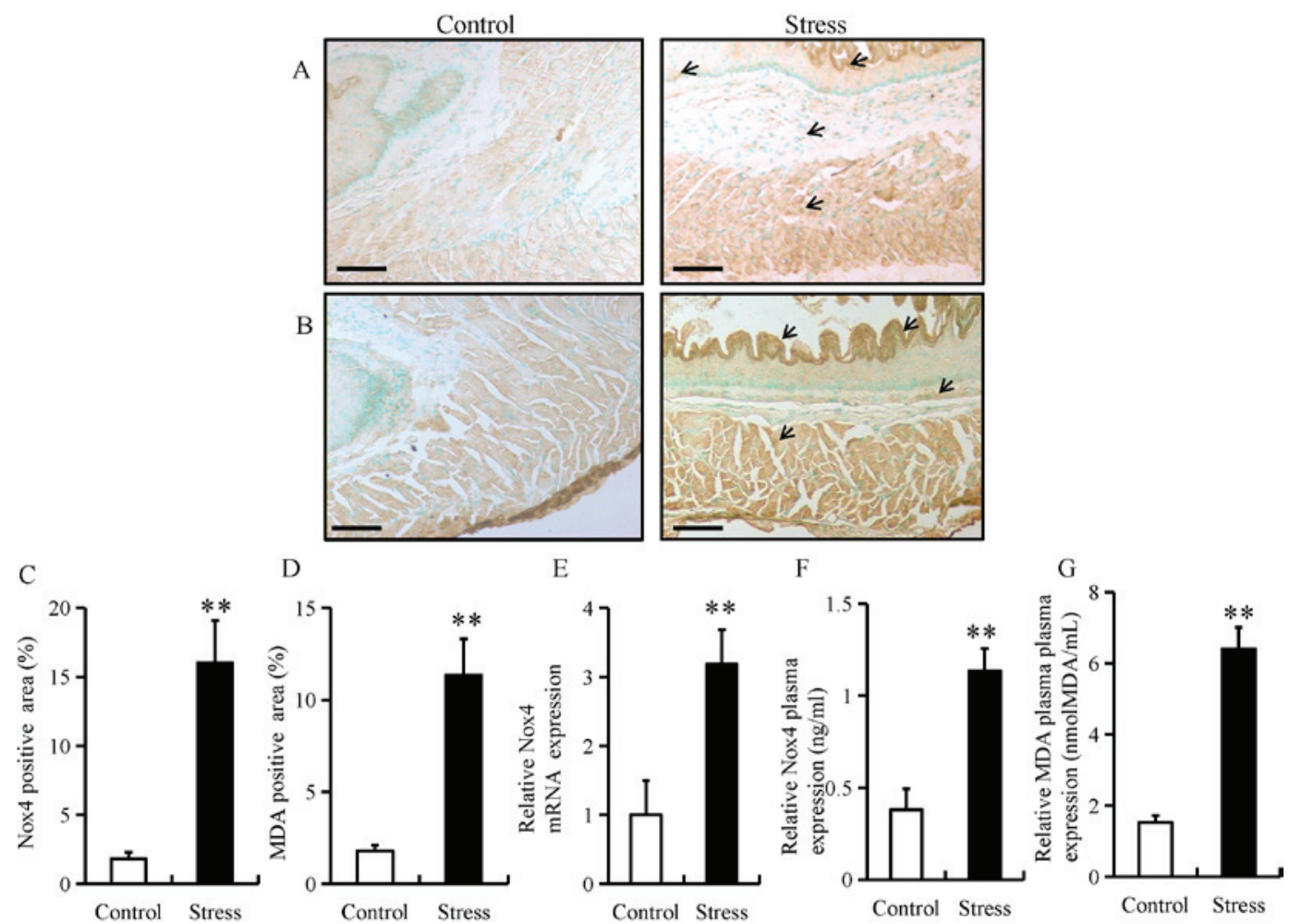

Figure 3. Chronic restraint stress induces ROS generation in the esophagus. Immunostaining of (A) Nox4 and (B) MDA in esophageal tissue from mice in the chronic restraint stress and control groups (magnification, x200; scale bar, $50 \mu \mathrm{m}$ ). Quantification of (C) Nox4 and (D) MDA positive areas. (E) The relative mRNA expression level of Nox4 was determined by reverse transcription-quantitative PCR in esophageal tissue from mice in the chronic restraint stress and control groups. (F) Nox4 and (G) MDA expression was determined by ELISA using plasma samples from mice in the chronic restraint stress and control groups. Data presented as the mean \pm standard deviation $(n=15) .{ }^{* *} \mathrm{P}<0.001$ vs. control. ROS, reactive oxygen species; Nox4, NAPDH oxidase 4; MDA, malondialdehyde. 

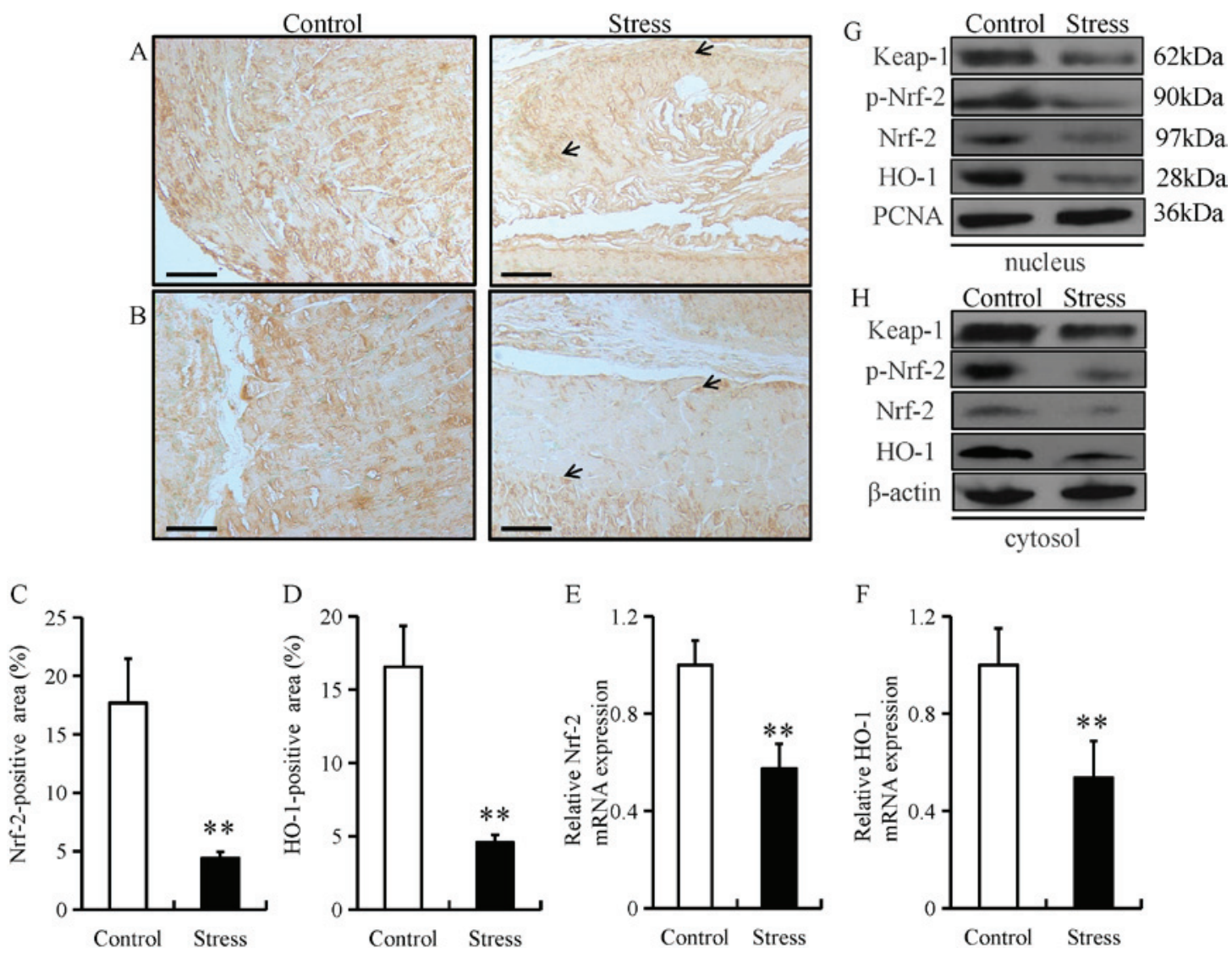

Figure 4. Chronic restraint stress reduces esophageal expression of anti-oxidant proteins. Immunostaining of (A) Nrf-2 and (B) HO-1 in esophageal tissue from mice in the chronic restraint stress and control groups (magnification, x200; scale bar. $50 \mu \mathrm{m}$ ). Quantification of the (C) Nrf-2 and (D) HO-1 positive areas The relative mRNA expression of (E) Nrf-2 and (F) HO-1 was determined by reverse transcription-quantitative PCR in esophageal tissue from mice in the chronic restraint stress and control groups. The relative protein expression of Keap-1, p-Nrf-2, Nrf-2 and HO-1 in the (G) nucleus and (H) cytosol fractions were determined by western blot analysis in esophageal tissue from mice in the chronic restraint stress and control groups. Data presented as the mean \pm standard deviation ( $\mathrm{n}=15) .{ }^{* *} \mathrm{P}<0.001$ vs. control. Keap1, Kelch like ECH associated protein 1; Nrf-2, nuclear factor erythroid 2-related factor 2; p-, phosphorylated; HO-1, heme oxygenase 1.

differences in the food intake, cholesterol or triglyceride levels of mice in the chronic restraint stress group compared with the control group (Table II). However, the concentration of FFA was significantly increased in the chronic restraint stress group compared with the control group (Table II).

Chronic restraint stress induces esophageal fibrosis in mice. To examine stress-induced histopathological changes and fibrosis, esophageal tissue from mice in the chronic restraint stress and control groups were stained with H\&E, Sirus red or MT. H\&E staining demonstrated that chronic restraint stress increased lymphocytic infiltration (as indicated by arrows) and the degree of inflammation within the submucosa of the esophagus, while there were no distinct changes observed in the control group (Fig. 5A and E). Furthermore, MT and sirus red staining highlighted the occurrence of fibrosis (as indicated by arrows) in the esophagus of the stressed mice (Fig. 5B, C and F). The MT-positive fibrotic areas in esophageal tissue from mice in the chronic restraint stress group were increased compared with the control group (Fig. 5F). IHC for F4/80 (specific for monocytes/macrophages, respectively) was performed in esophageal tissue from mice in the chronic restraint stress and control groups. The results demonstrated that $\mathrm{F} 4 / 80$ expression was predominantly located in the mucosal and epithelial layers of the esophagus (as indicated by arrows), and that expression levels significantly increased in the chronic restraint stress group compared with the control group (Fig. 5D and G).

\section{Discussion}

The current study identified several key findings. The expression of fibrotic proteins, including collagen type I, TGF- $\beta 1$, SMAD-3 and $\alpha$-SMA, demonstrated that chronic restraint stress may induce esophageal fibrosis in mice. In addition, chronic restraint stress may induce oxidative stress as the expression levels of Nox4 and MDA were significantly increased in mice. Furthermore, chronic restraint stress reduced the expression of several anti-oxidative proteins, including Keap-1, p-Nrf-2, Nrf-2 and HO-1 in the esophageal tissue of mice. In conclusion, the current study demonstrated that chronic stress may trigger esophageal fibrosis by enhancing oxidative stress and suppressing the anti-oxidative system.

Oxidative stress is an essential factor in the pathogenesis of esophageal injury/repair and in esophageal barrier dysfunction (25). NADPH oxidases, including Nox4, are the main producers of ROS in the esophagus and serve key roles in esophageal remodeling, esophageal barrier dysfunction and inflammation $(26,27)$. Previous studies have demonstrated that two weeks of chronic restraint stress can trigger inflammation and ROS accumulation in different types of tissues $(18,20)$. 
Table II. Chronic restraint stress-induced weight loss and FFA release in mice.

\begin{tabular}{lrrr}
\hline & \multicolumn{1}{c}{ Control } & \multicolumn{1}{c}{ Stress } & P-value \\
\hline BW gain $(\mathrm{g})$ & $1.37 \pm 0.02$ & $1.04 \pm 0.04$ & $<0.001$ \\
Food intake $(\mathrm{mg})$ & $133.5 \pm 2.46$ & $130.7 \pm 2.46$ & 0.441 \\
TC $(\mathrm{mg} / \mathrm{dl})$ & $64 \pm 2.42$ & $70.6 \pm 3.23$ & 0.123 \\
TG $(\mathrm{mg} / \mathrm{dl})$ & $8.19 \pm 0.39$ & $8.95 \pm 0.62$ & 0.324 \\
FFA (mEq/l) & $0.36 \pm 0.03$ & $0.91 \pm 0.05$ & $<0.001$ \\
\hline
\end{tabular}

Data presented as the mean \pm SD $(n=15)$. BW, body weight; TC, total cholesterol; TG, tryglycerides; FFA, free fatty acids.
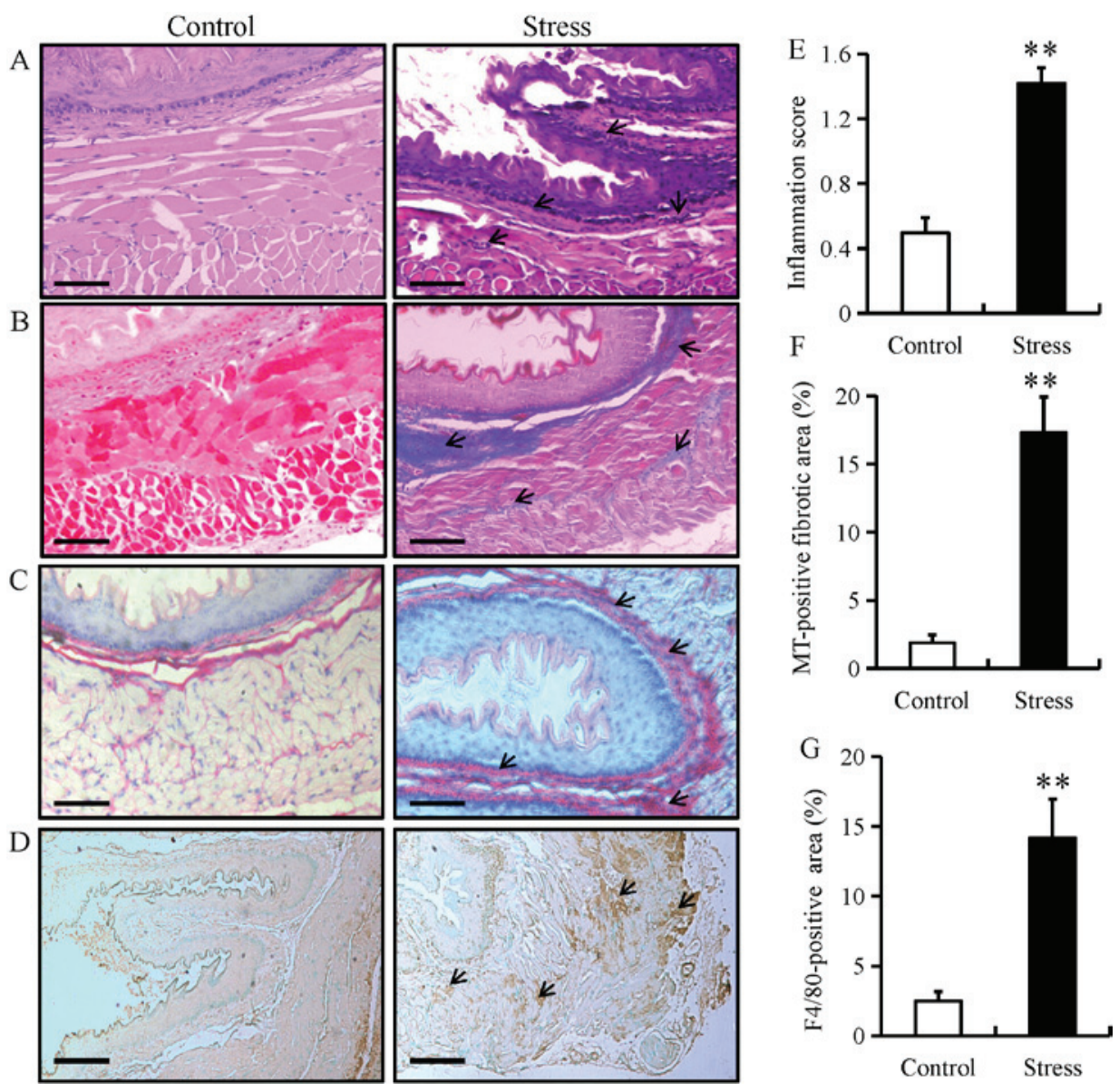

Figure 5. Chronic restraint stress induces esophageal fibrosis in mice. Esophageal tissue from mice in the chronic restraint stress and control groups were analyzed by (A) H\&E, (B) MT and (C) Sirus red staining. (magnification, x200; scale bar, $50 \mu \mathrm{m}$ ). (D) Immunostaining for F4/80 in esophageal tissue from mice in the chronic restraint stress group and control group (magnification, x200; scale bar, $50 \mu$ m). (E) Histology inflammation score. (F) Quantification of the MT-positive areas. (G) Quantification of the F4/80-positive areas. Data presented as the mean \pm standard deviation ( $\mathrm{n}=15$ ). ${ }^{* *} \mathrm{P}<0.001 \mathrm{vs}$. control. H\&E, hematoxylin and eosin; MT, Masson's trichrome.

Oxidative stress also leads to esophageal fibrosis by increasing the expression of TGF- $\beta 1$, which enhances the synthesis of esophageal collagen and suppresses the degradation of collagen in the gastroesophageal reflux disease (GERD) model $(25,28)$. The current study demonstrated that chronic restraint stress enhanced the in vivo esophageal expression of Nox4 and MDA, a biomarker of oxidative stress.

Chronic psychological stress can induce oxidative stress in different tissues, including the brain and peripheral blood cells, and these adverse effects can be partially reversed by anxiolytic agents (29). A previous study demonstrated that two weeks of chronic restraint stress in mice caused an accumulation of ROS and inflammation in several types of tissue, including visceral adipose tissue (VAT) as well as liver and intestine (21). Suppressed chronic stress-induced ROS production and VAT inflammation were identified as potential therapeutic targets for stress-associated disorders (18). Increased ROS accumulation in VAT is accompanied by increased NADPH oxidase (NOX) subunits and decreased antioxidant enzymes and has been recognized as an early marker and potential therapeutic target of metabolic syndrome (16). 
Activated myofibroblasts are key effector cells in all models of fibrosis. In wound healing, tissue strain and cytokine release activate myofibroblasts, which initiate migration, extracellular matrix (ECM) deposition and tissue contraction, thereby maintaining tissue homeostasis (30). However, in fibrosis, an exaggerated myofibroblast response results in inappropriate ECM deposition, increased tissue stiffness and organ dysfunction (31). As epithelial cells are capable of transdifferentiation under these conditions, it has been recognized that during chronic inflammation, epithelial cells undergo epithelial-to-mesenchymal transition in fibrosis (32). As the epithelium is often the site of primary injury and inflammation, epithelial cells may also function as effector cells in fibrogenesis.

Oxidative stress is closely associated with the pathogenesis of GERD, which leads to increased ROS production (33). Long-term exposure to oxidative stress in GERD induces chronic inflammation and fibrosis in the esophagus, which leads to the formation and progression of disease states in esophageal tissue (25). In addition, markers for oxidative stress are overexpressed in patients with GERD, which indicates that increased ROS may be primarily responsible for the development of GERD (34). ROS also leads to esophageal fibrosis by increasing the expression of TGF- $\beta 1$, which enhances the synthesis of esophageal collagen and suppresses the degradation of collagen in the GERD model (35). In the present study, chronic restrain stress upregulated subunits of NOX, a major source of ROS and downregulated antioxidant proteins in the esophagus.

In the current study, direct measurement of ROS was not performed and this may be considered a limitation associated with the study, which will need to be addressed in future work. In a previous study, chronic restraint stress markedly induced the accumulations of ROS in adipose (18) and colon tissue (21). The present study examined the expression levels of ROS markers including, Nox4 and MDA. The results demonstrated that two weeks of chronic restraint stress significantly increased the expression of Nox 4 and MDA in the mucosal and epithelial layers of the esophagus. In addition, mRNA and plasma levels of Nox4 and MDA were significantly increased in the esophageal tissue of mice in the chronic restraint stress group compared with the control group. Taken together, these results indicate that chronic stress significantly increased ROS production in the esophagus of mice.

The Nrf-2/Keap-1 signaling pathway provides cells with a defense mechanism against oxidative stress by regulating the expression of enzymes that serve key roles in the anti-oxidative stress response and detoxification (36). Esophageal hyperkeratosis in Keap-1 knockout mice was due to activation of peroxisome proliferator-activated receptor- $\beta / \Delta$ and the PI3K/Akt pathway (37). Chen et al (38) demonstrated that Nrf-2 deficiency impairs the barrier function of mouse esophageal epithelium by disrupting the expression of tight junction proteins. Furthermore, as a downstream gene of $\mathrm{Nrf}-2, \mathrm{HO}-1$ prevents gastroesophageal reflux-induced esophageal barrier dysfunction by suppressing oxidative stress in mouse models of GERD (38). In summary, the present study demonstrated that chronic stress reduced the esophageal expression of several anti-oxidative proteins including, Keap-1, phopho-Nrf-2, Nrf-2 and HO-1 in mice.

TGF- $31 /$ SMAD-3 signaling has been recognized as a common pathway involved in several fibrotic diseases (39). TGF- $\beta 1$ overexpression can introduce several negative impacts, which include promoting direct transcription of pro-fibrotic factors, such as collagen type I, and the contraction of cultured esophageal smooth muscle cells (40). Cells secrete TGF- $\beta 1$, which binds to TGF- $\beta 1$ cell surface receptors (TGF- $\beta$ RI and TGF- $\beta$ RII) on fibroblasts. These receptors can activate and translocate intracellular SMADs (a family of transcription factors that mediate TGF- $\beta 1$ signals) to the nucleus, where they regulate transcription of collagen genes, which contributes to fibrosis (26). The phosphorylation of the receptor-dependent SMAD2/3, along with SMAD4, creates a complex, which can be translocated from the cytoplasm to the nucleus to regulate collagen gene transcription (28).

Previous studies have demonstrated several types of SMAD-3-dependent collagen gene promoters, which are activated by TGF- $\beta 1(41,42)$. Dominant-negative SMAD-3 expression vectors specifically inhibited the activation of these promoters (43). Cho et al (44) examined the role of SMAD-3 in a mouse model of egg-induced eosinophilic esophagitis and revealed that SMAD-3-deficient mice esophageal fibroblasts could not respond to TGF- $\beta 1$ to regulate the expression of collagen genes, thereby reducing esophageal fibrosis. However, in the current study, chronic stress increased the esophageal expression of fibrotic genes in mice.

In conclusion, two weeks of chronic restraint stress induced esophageal fibrosis in a murine model with enhanced oxidative stress and reduced the anti-oxidative system.

\section{Acknowledgements}

The authors would like to thank Dr Kyosuke Takeshita for carefully reading and editing to the manuscript.

\section{Funding}

The current study was supported by a grant from the Xinjiang Uygur Autonomous Region Natural Science Foundation Program (grant no. 2018D01C134).

\section{Availability of data and materials}

All data that generated or analyzed during this study are included in this published article.

\section{Authors' contribution}

MY and KA designed the current study, performed the experiments and prepared the manuscript. WW, AiA, YL, AzA, AlA, MA, WZ, ZC and AM performed the experiments, collected and analyzed the data, and prepared the manuscript. All authors read and approved the final manuscript.

\section{Ethics approval and consent to participate}

All animal experiments were approved by the Animal Care and Use Committee of the People's Hospital of Xinjiang Uygur Autonomous Region (Urumqi, China).

\section{Patient consent for publication}

Not applicable. 


\section{Competing interests}

The authors declare that they have no competing interests.

\section{References}

1. Godoy LD, Rossignoli MT, Delfino-Pereira P, Garcia-Cairasco N and de Lima Umeoka EH: A comprehensive overview on stress neurobiology: Basic concepts and clinical implications. Front Behav Neurosci 12: 127, 2018.

2. Liu YZ, Wang YX and Jiang CL: Inflammation: The common pathway of stress-related diseases. Front Hum Neurosci 11: 316, 2017.

3. Lucca G, Comim CM, Valvassori SS, Reus GZ, Vuolo F, Petronilho F, Dal-Pizzol F, Gavioli EC and Quevedo J: Effects of chronic mild stress on the oxidative parameters in the rat brain. Neurochem Int 54: 358-362, 2009.

4. Tomanek L: Proteomic responses to environmentally induced oxidative stress. J Exp Biol 218: 1867-1879, 2015.

5. Gutierrez J, Ballinger SW, Darley-Usmar VM and Landar A: Free radicals, mitochondria, and oxidized lipids: The emerging role in signal transduction in vascular cells. Circ Res 99: 924-932, 2006.

6. Zafir A and Banu N: Modulation of in vivo oxidative status by exogenous corticosterone and restraint stress in rats. Stress 12: $167-177,2009$.

7. Gumuslu S, Sarikçioğlu SB, Sahin E, Yargiçoğlu P and Ağar A: Influences of different stress models on the antioxidant status and lipid peroxidation in rat erythrocytes. Free Radic Res 36 1277-1282, 2002.

8. Sahin E and Gümüşlü S: Immobilization stress in rat tissues: Alterations in protein oxidation, lipid peroxidation and antioxidant defense system. Comp Biochem Physiol C Toxicol Pharmacol 144: 342-347, 2007.

9. Li-Kim-Moy JP, Tobias V, Day AS, Leach S and Lemberg DA: Esophageal subepithelial fibrosis and hyalinization are features of eosinophilic esophagitis. J Pediatr Gastroenterol Nutr 52: 147-153, 2011.

10. Linares V, Sánchez DJ, Bellés M, Albina L, Gómez M and Domingo JL: Pro-oxidant effects in the brain of rats concurrently exposed to uranium and stress. Toxicology 236: 82-91, 2007.

11. Bedard K and Krause KH: The NOX family of ROS-generating NADPH oxidases: Physiology and pathophysiology. Physiol Rev 87: 245-313, 2007.

12. Babalola O, Mamalis A, Lev-tov $\mathrm{H}$ and Jagdeo J: NADPH oxidase enzymes in skin fibrosis: Molecular targets and therapeutic agents. Arch Dermatol Res 306: 313-330, 2014

13. Hecker L, Cheng J and Thannickal VJ: Targeting NOX enzymes in pulmonary fibrosis. Cell Mol Life Sci 69: 2365-2371, 2012.

14. De Minicis S and Brenner DA: NOX in liver fibrosis. Arch Biochem Biophys 462: 266-272, 2007.

15. Pennathur S, Hecker L and Thannickal VJ: Oxidative Stress and Cardiovascular Fibrosis: Humana Press: pp425-441, 2010.

16. Holterman CE, Read NC and Kennedy CR: Nox and renal disease. Clin Sci (Lond) 128: 465-481, 2015.

17. Yisireyili M, Hayashi M, Wu H, Uchida Y, Yamamoto $\mathrm{K}$, Kikuchi R, Shoaib Hamrah M, Nakayama T, Wu Cheng X, Matsushita T, et al: Xanthine oxidase inhibition by febuxostat attenuates stress-induced hyperuricemia, glucose dysmetabolism and prothrombotic state in mice. Sci Rep 7: 1266, 2017.

18. Yisireyili M, Takeshita K, Hayashi M, Wu H, Uchida Y, Yamamoto K, Kikuchi R, Hao CN, Nakayama $T$, Cheng XW, et al: Dipeptidyl peptidase-IV inhibitor alogliptin improves stress-induced insulin resistance and prothrombotic state in a murine model. Psychoneuroendocrinology 73: 186-195, 2016.

19. Li Q, Kong L, Zhang S, Zhong Z, Liu X, Wang J and Kang J: A novel external esophageal perfusion model for reflux-associated respiratory symptoms. Pathobiology 77: 163-168, 2010.

20. Livak KJ and Schmittgen TD: Analysis of relative gene expression data using real-time quantitative PCR and the 2(-Delta Delta C(T)) method. Methods 25: 402-408, 2001.

21. Yisireyili M, Uchida Y, Yamamoto K, Nakayama T, Cheng XW, Matsushita T, Nakamura S, Murohara T and Takeshita K: Angiotensin receptor blocker irbesartan reduces stress-induced intestinal inflammation via AT1a signaling and ACE2-dependent mechanism in mice. Brain Behav Immun 69: 167-179, 2018.
22. Wakabayashi N, Itoh K, Wakabayashi J, Motohashi H, Noda S, Takahashi S, Imakado S, Kotsuji T, Otsuka F, Roop DR, et al: Keap1-null mutation leads to postnatal lethality due to constitutive Nrf2 activation. Nat Genet 35: 238-245, 2003.

23. Torihata Y, Asanuma K, Iijima K, Mikami T, Hamada S, Asano N, Koike T, Imatani A, Masamune A and Shimosegawa T: Estrogen-dependent Nrf2 expression protects against reflux-induced esophagitis. Dig Dis Sci 63: 345-355, 2018.

24. Peng D, Belkhiri A, Hu T, Chaturvedi R, Asim M, Wilson KT,Zaika $\mathrm{A}$ and El-Rifai W: Glutathione peroxidase 7 protects against oxidative DNA damage in oesophageal cells. Gut 61: 1250-1260, 2012.

25. Oh TY, Lee JS, Ahn BO, Cho H, Kim WB, Kim YB, Surh YJ, Cho SW and Hahm KB: Oxidative damages are critical in pathogenesis of reflux esophagitis: Implication of antioxidantsin its treatment. Free Radic Biol Med 30: 905-915, 2001.

26. Aceves SS: Remodeling and fibrosis in chronic eosinophil inflammation. Dig Dis 32: 15-21, 2014.

27. Wynn TA and Ramalingam TR: Mechanisms of fibrosis: Therapeutic Translation for fibrotic disease. Nat Med 18: 1028-1040, 2012

28. Sziksz E, Pap D, Lippai R, Béres NJ, Fekete A, Szabó AJ and Vannay Á: Fibrosis related inflammatory mediators: Role of the IL-10 cytokine family. Mediators Inflamm 2015: 764641, 2015.

29. Miller MW and Sadeh N: Traumatic stress, oxidative stress and post-traumatic stress disorder: Neurodegeneration and the accelerated-aging hypothesis. Mol Psychiatry 19: 1156-1162, 2014.

30. Frangogiannis NG: Fibroblast-extracellular matrix interactions in tissue fibrosis. Curr Pathobiol Rep 4: 11-18, 2016.

31. Hosper NA, van den Berg PP, de Rond S, Popa ER, Wilmer MJ, Masereeuw R and Bank RA: Epithelial-to-mesenchymal transition in fibrosis: Collagen type I expression is highly upregulated after EMT, but does not contribute to collagen deposition. Exp Cell Res 319: 3000-3009, 2013.

32. Hinz B, McCulloch CA and Coelho NM: Mechanical regulation of myofibroblast phenoconversion and collagen contraction. Exp Cell Res 379: 119-128, 2019.

33. Song JH, Han YM, Kim WH, Park JM, Jeong M, Go EJ, Hong SP and Hahm KB: Oxidative stress from reflux esophagitis to esophageal cancer; the alleviation with antioxidants. Free Radic Res 50: 1071-1079, 2016.

34. Dandekar A, Mendez R and Zhang K: Cross talk between ER stress, oxidative stress, and inflammation in health and disease. Methods Mol Biol 1292: 205-214, 2015.

35. Maqbool A and Pauwels A: Cystic fibrosis and gastroesophageal reflux disease. J Cyst Fibros 2 (16 Suppl): S2-S13, 2017.

36. Jaramillo MC and Zhang DD: The emerging role of the Nrf2-Keap1 signaling pathway in cancer. Genes Dev 27: 2179-2191, 2013.

37. Chen H, Fang Y, Li W, Orlando RC, Shaheen N and Chen XL: NFkB and Nrf2 in esophageal epithelial barrier function. Tissue Barriers 1: e27463, 2013.

38. Chen H, Hu Y, Fang Y, Djukic Z, Yamamoto M, Shaheen NJ, Orlando RC and Chen X: Nrf2 deficiency impairs the barrier function of mouse oesophageal epithelium. Gut 63: 711-719, 2014.

39. Balta C, Herman H, Boldura OM, Gasca I, Rosu M, Ardelean A and Hermenean A: Chrysin attenuates liver fibrosis and hepatic stellate cell activation through TGF- $\beta /$ Smad signalingpathway. Chem Biol Interact 240: 94-101, 2015.

40. Kuppan P, Sethuraman S and Krishnan UM: In vitro co-culture of epithelial cells and smooth muscle cells on aligned nanofibrous scaffolds. Mater Sci Eng C Mater Biol Appl 81: 191-205, 2017.

41. Ghosh AK, Bhattacharyya S and Varga J: The tumor suppressor p53 abrogates Smad-dependent collagen gene induction in mesenchymal cells. J Biol Chem 279: 47455-47463, 2004.

42. Ito S, Ogawa K, Takeuchi K, Takagi M, Yoshida M, Hirokawa T, Hirayama S, Shin-Ya K, Shimada I, Doi T, et al: A small-molecule compound inhibits a collagen-specific molecular chaperone and could represent a potential remedy for fibrosis. J Biol Chem 292: 20076-20085, 2017.

43. Hu Z, Robbins JS, Pister A, Zafar MB, Zhang ZW, Gupta J, Lee KJ, Newman K, Yun CO, Guise T and Seth P: A modified hTERT promoter-directed oncolytic adenovirus replication with concurrent inhibition of TGFbeta signaling for breast cancer therapy. Cancer Gene Ther 17: 235-243, 2010.

44. Cho JY, Doshi A, Rosenthal P, Beppu A, Miller M, Aceves S and Broide D: Smad3-deficient mice have reduced esophageal fibrosis and angiogenesis in model of egg-induced eosinophilic esophagitis. J Pediatr Gastroenterol Nutr 59: 10-16, 2014. 